\section{GABRIELLA ZARRI}

Univ. Firenze, Dipartimento di Scienze dell'Antichità, Medioevo e Rinascimento e Linguistica

\section{Paolo Prodi}

e gli studi storici negli anni '60 e '70
STORICAMENTE

Rivista del Dipartimento di

Storia Culture Civiltà

Alma Mater Studiorum

Università di Bologna

DOI: $10.12977 /$ stor488

ISSN: $1825-411 X$

Art. No. 35

Issue No 9 - 2013

Publisher: BraDypuS

July $24^{\text {th }} 2013$

Dibattiti - Storia moderna 0 genesi della modernità? Dibattito in occasione degli 80 anni di Paolo Prodi

Nessun libro come quello di cui oggi si parla si presta in maniera migliore a presentare la figura di un maestro come Paolo Prodi. Sono qui raccolti in sintesi alcuni degli interventi più significativi riguardanti la disciplina che ha scelto e praticato per lunghi anni impartendo un insegnamento che è sempre stato affiancato e sorretto dall'impegno culturale e politico, alternando, come si è sempre premurato di fare, il tempo dedicato alla ricerca e allo studio con quello rivolto alla gestione delle strutture universitarie $\mathrm{o}$ alla creazione e rivitalizzazione di società scientifiche. Un impegno che gli ha consentito di approfondire le originali riflessioni storiche che gli sono proprie e di vivere intensamente il proprio tempo, orientando e rinnovando gli indirizzi di lavoro sulla base dei problemi e delle discussioni emergenti.

Non è un caso che un traguardo personale ragguardevole come quello dell'ottantesimo compleanno sia festeggiato con la presentazione di un libro dello stesso Paolo Prodi, che mostra con la sua inesauribile vitalità di essere ben presente nella scena culturale e politica di questo paese e di avere una posizione precisa riguardo a temi fondamentali per la disciplina storica e per il contesto culturale attuale.

Di questo libro vorrei soltanto sottolineare due cose: la prima è la persistenza pluridecennale di una riflessione sulla storia e sulla metodologia storica che non propone invariabilmente se stessa, ma che si arricchisce continuamente del dibattito storiografico in atto contribuendo in modo determinante ad orientarlo.

La seconda è la straordinaria capacità di questo libro di riflettere la personalità dell'autore. Come, mi si dirà, una raccolta di saggi è più significativa di una delle 
ciclopiche ricerche storiche del Paolo Prodi degli ultimi anni? Ebbene sì, direbbe un amico comune: da un nuovo libro ci si aspetta sempre qualcosa di originale e di innovativo. Da una raccolta di saggi no. Ebbene quel genere storiografico che Prodi ha sempre aborrito, la raccolta di saggi, è qui divenuto opera originale. Specchio del lavoro di un maestro, palestra di metodo per uno studente che si accosta oggi alla storia.

Mai come in questo libro ho trovato posizioni ferme e chiarificatrici sulla questione della modernità: discussione certo metodologica ma anche fortemente ideologica che ha convogliato sul dibattito illuminismo/rivoluzione quell'opposizione che per più di un secolo ha polarizzato il dibattito riforma protestante progressiva /controriforma regressiva. Il ricorso ad una frase di Voltaire per orientare e rafforzare la datazione della modernità al secolo XV/XVI mi è sembrata una mossa da maestro, ma quello che appare più convincente è il richiamo alla forza determinante della continuità storica che prevale e avvolge, preparandola e poi inglobandola, sulla discontinuità e sulla frattura rivoluzionaria. La metafora del terremoto è più che mai attuale.

E vi è anche nel libro un'altra metafora che mi ha colpito e che rispecchia in modo efficace la personalità dell'autore, alieno dagli intellettualismi di parte della categoria e propenso alle cose concrete e alla quotidianità: la metafora della scatola. Nel libro, che ripropone un saggio del 2006 dedicato a storia moderna e società contemporanea, Paolo Prodi spiega al nipotino Francesco la differenza tra antico e moderno e gli parla di scatole in cui noi cerchiamo di mettere in ordine, di organizzare ciò che il passato ci ha lasciato. Illustra poi gli aspetti caratterizzanti della disciplina storica enucleandoli in quattro punti: la scatola temporale, la scatola spaziale, gli strumenti, i contenuti. Conclude poi con la constatazione della dissoluzione delle categorie che abbiamo fin qui considerato costitutive del moderno, come la centralità del progresso, la crisi del monopolio instaurato dalla stato nazione e il pericolo di una retrocessione ad una identificazione etnica o di tipo religioso-ideologico. Di qui la necessità di scongiurare il ritorno di fondamentalismi razziali e religiosi con la ricostruzione di identità collettive come articolazioni complesse.

Ma non voglio addentrarmi sulle riflessioni e proposte che hanno animato le ricerche collettive degli ultimi anni di Paolo Prodi e di coloro che gli erano più vicini. Da vecchia allieva pensionanda ricordo invece volentieri i primi tempi dell'insegnamento di Paolo Prodi, quando le scatole della storia erano quelle delle scarpe, come si addiceva ad una società ancora povera e priva dei mezzi tecnologici che di anno in anno hanno reso più facile e più efficiente il lavoro di ricerca e di catalogazione delle fonti storiche e delle opere letterarie. 
Dunque mi considero qui soprattutto come testimone di una avventura intellettuale iniziata in tempi lontani, quando alla crisi politico sociale del dopoguerra seguiva un tempo di espansione economico demografica che favoriva l'allargarsi dell'Istituzione universitaria e il suo aprirsi a nuovi soggetti sociali. Era il tempo della fondazione dei Magisteri, del più esteso accesso alle donne dell'istruzione universitaria, delle prime battaglie per il riconoscimento dei diritti civili alle donne. In Università convivevano le antitetiche figure dell'assistente volontario, intelligente e colto figlio di famiglia, del professore di scuola media superiore che continuava la tradizione della vecchia scuola social liberale e massonica ottocentesca per cui si accedeva al vertice delle Istituzioni culturali dopo aver percorso un lungo cammino di studio che prevedeva dopo la laurea una libera docenza acquisita per lo più dopo l'accesso al lavoro di insegnamento o altra attività remunerativa, c'erano infine alcuni assistenti cui era consentito per concorso nazionale di accedere direttamente all'insegnamento universitario. Paolo Prodi era uno di questi.

Era il 1963 quando sostenni con lui l'esame di storia medievale, un anno che si era aperto nel segno di una grande speranza e che si concludeva in modo traumatico e drammatico. Nel 1962 l'inaugurazione del Concilio ecumenico Vaticano II che vedeva la riunione di oltre 2000 padri conciliari provenienti da tutte le parti del mondo assurgeva a segno simbolico di pacificazione universale, dopo gli orrori e le distruzioni della guerra mondiale, la divisione del mondo in blocchi contrapposti e armati, l'apparente impossibilità dell'Italia di divenire, dopo le lotte e le divisioni della guerra e del dopoguerra, "un paese normale". L'America di John Kennedy, economicamente in crescita, avviava processi di riconoscimento di diritti umani e civili verso la popolazione nera e gli stranieri e il superamento diplomatico della "crisi" di Cuba apriva un periodo di distensione inatteso, date le tesissime relazioni delle due superpotenze mondiali. Nell'autunno del 1963 si realizzò in Italia il primo centro sinistra, con un governo presieduto da Aldo Moro e da Pietro Nenni in qualità di vice-presidente. Ma in novembre l'assassinio di John Kennedy riproponeva in tutta la sua spettacolare teatralità la persistenza dei molteplici conflitti in atto e assumeva il significato della fine di un sogno, potremmo dire tipicamente americano, di benessere e pace universale. Restava tuttavia il Concilio ecumenico che, dopo le scomuniche moderniste di inizio secolo, si apriva al mondo moderno e rappresentava nel contesto culturale di quegli anni una promessa di profonde trasformazioni.

Chi viveva e studiava a Bologna in quegli anni ed era interessato a seguire questo evento con consapevolezza critica aveva nel Centro di Documentazione. Istituto per le scienze religiose, fondato a metà degli anni Cinquanta da don Giuseppe 
Dossetti, e allora diretto da Giuseppe Alberigo, la possibilità di approfondire teologicamente e storicamente le questioni trattate. Non solo: chi trascorreva ore e giorni in quell'Istituto aveva la netta percezione di trovarsi nella cucina del Concilio. Non perché si arrotassero i coltelli, ma perché le più gustose vivande erano preparate da cuochi provetti. In poco tempo, con un lavoro di equipe che dette subito duraturi ed ottimi risultati, venne sfornato un aureo strumento di lavoro per gli studiosi e i padri conciliari: i Conciliorum oecumenicorum decreta, edizione critica di tutti i Concili della Chiesa cristiana che sostituiva un precedente parziale Enchiridion e si affiancava con agile maneggiabilità ai molti e molti volumi dell'erudizione settecentesca. Tra quei cuochi c'era anche Paolo Prodi che convertiva temporaneamente il suo interesse e vocazione politica allo studio dei problemi della chiesa nei suoi rapporti con il mondo moderno, cercando nella storia le radici dell'assetto teologico e istituzionale di quella chiesa che Giovanni XXIII si proponeva di aggiornare, analizzando al tempo stesso le dottrine e le vicende che avevano posto per secoli il dominio temporale della chiesa al centro della politica italiana ed europea. Come Paolo Prodi rispondesse allora e come rispose successivamente ad una domanda che rimase la stella polare della sua futura ricerca è sotto gli occhi di tutti Ovviamente sono gli studi individuali quelli a cui è affidata in prima linea l'influenza culturale e intellettuale di Paolo Prodi in Italia e nel mondo, ma i lavori personali non esauriscono l'impegno scientifico che il professore ha profuso nella sua attività, con costanza, metodicità e senza risparmio di energie.

Come testimone della prima ora di quel lavoro vorrei brevemente ricordare l'attività didattica di Paolo Prodi nel periodo del suo primo insegnamento bolognese, quello che va dal 1963 al 1973 circa: un afflusso sempre maggiore di studenti agli studi universitari; una domanda di trasformazione politica e liberalizzazione degli studi che culmina nei movimenti studenteschi del 1968 e avvia processi culturali la cui portata si avvertì immediatamente nella compagine universitaria $\mathrm{e}$ ben presto anche nel costume e nella società. A fronte di questa irrequietezza culturale che era in stretto rapporto con il trend positivo dell'economia - quel boom economico che si manifestava allora anche in Italia - corrispondeva la resistenza al cambiamento opposta talvolta dai docenti, qualche altra volta dalle mancanze di strutture e spazi materiali per gli studi e la ricerca. Per non parlare della limitatezza allora dei mezzi tecnologici e di comunicazione, come prima si diceva. Varrà forse la pena ricordare che quando il prof. Prodi mi propose un titolo per la tesi, mi avviò alla ricerca in un modo piuttosto singolare. Mi trovavo in quel Centro di Documentazione dove si lavorava alacremente per il Concilio e al tempo stesso si arricchiva progressivamente la biblioteca di libri di storia, teologia, 
esegesi biblica, spiritualità e Prodi lavorava al suo libro sul Cardinal Paleotti dividendo il suo tempo tra studio e archivio. Mi mostrò i primi strumenti o meglio supporti del lavoro di ricerca: due bianche schede di diversa dimensione, una più piccola per la raccolta delle informazioni bibliografiche e una più grande, di una dimensione fuori commercio e fatta tagliare artigianalmente in quantità industriali. La misura della scheda era quella che corrispondeva alla larghezza e altezza di una scatola da scarpe, sufficientemente grande per copiare parte di documenti e adatta ad essere custodita e ordinata in contenitori per niente costosi. Insieme al successivo insegnamento di Gina Fasoli che il lavoro dello storico è di forbici e colla, mi accinsi ad affrontare la ricerca nella convinzione che si trattasse di un lavoro artigianale che si svolgeva in tranquilli e quieti ambienti che custodivano memorie e testimonianze del passato, ricercate e interpretate alla luce del presente. Non ci volle molto tempo perché la mia ingenuità venisse messa alla prova, ma di questo non parlerò.

Ho parlato fin qui di un rapporto personale, fuori di quegli spazi accademici dove durante gli studi non mi recavo molto spesso. In quegli anni infatti, siamo nel 1965, il giovane Paolo Prodi non aveva molti studenti e non aveva quindi ancora messo in atto quello strumento che considero il più riuscito mezzo di formazione didattica che egli abbia mai attuato: il seminario dei laureandi. Se non potei beneficiare da studentessa del seminario, perché lavoravo come insegnante, ebbi però l'occasione di ascoltare le lezioni di due storici che ebbero molta influenza nel mio successivo lavoro e che Prodi aveva invitato appositamente per coloro che avevano iniziato la tesi: Hubert Jedin e Marino Berengo. Di Jedin è inutile dire, tanto era di casa a Bologna in quegli anni. Di Marino Berengo dirò che, in procinto di pubblicare la sua importante opera su Lucca, fece una lettura di alcune pagine di Botero tenendo una lezione sulle città repubblicane. Non so se voluto o occasionale, ma senza dubbio l'accostamento di due persone così diverse per interessi scientifici, tradizioni culturali, oggetto di studio costituì per me stimolo alla riflessione e rese evidente la necessità di tenere insieme aspetti molteplici della ricerca che, pur mossa da personali opzioni politiche e culturali, non ignora e valuta attentamente interpretazioni diverse.

Quando mi laureai, nel giugno del 1968, il corpo accademico aveva cominciato a rispondere al movimento studentesco svestendo la toga. La mia fu la prima seduta di laurea senza insegne del sapere/potere dei professori universitari. Paolo Prodi aveva vinto il concorso a cattedre e il Paleotti era fresco di stampa. Giungevano intanto da Pisa i suoi primi collaboratori nella didattica e nella ricerca. Adriano Prosperi fresco perfezionato della Scuola Normale e Serena Spanò. L'insegnamento si sdoppiava, cominciavano i seminari, aumentavano i laureandi. Fu allora 
che Prodi iniziò l'incontro settimanale dei laureandi, dedicato alla verifica del lavoro svolto da ciascuno, ma anche alla più estesa discussione dei problemi metodologici e storiografici connessi con le diverse tesi di ricerca. A turno ogni laureando la cui ricerca era ad un grado avanzato di elaborazione relazionava sul proprio tema di ricerca, prospettando le fonti e i problemi di interpretazione con queste connesse ed esponendo le linee direttive della sua indagine. Non tutti gli studenti avevano avuto l'insegnamento privilegiato che a me era stato riservato: il modello della scheda-scatola di scarpa, ma arrivavano comunque ricolmi di schede bibliografiche ed archivistiche, a contrassegnare una stagione degli studi di Paolo Prodi fortemente legata agli archivi. L'interesse del seminario consisteva soprattutto nel fatto che all'interno del gruppo vi erano diversi indirizzi di ricerca, che riproponevano ovviamente le tematiche allora vive negli studi del professare: ancora una volta la religione si intrecciava con la politica: vi era l'indirizzo religioso che privilegiava le indagini sulle istituzioni ecclesiastiche, le confraternite, le visite pastorali, l'applicazione del Concilio di Trento; l'indirizzo politicoistituzionale che spaziava dallo studio delle cronache cittadine all'analisi dei deliberati dei consigli comunali o delle magistrature cittadine, e al funzionamento della finanza pontificia; l'indirizzo culturale che prendeva in esame le istituzioni educative, le accademie e le Università; e infine quello più propriamente sociale, che comprendeva sia l'analisi degli istituti di assistenza e delle categorie marginali, sia l'esame dei problemi della giustizia connessi con la legislazione legatizia e con l'analisi di processi criminali.

La disciplina era ancora in espansione, così come l'afflusso degli studenti. Un nuovo docente, questa volta proveniente dall'Università cattolica, si affiancò a Paolo Prodi ad animare, se ce ne fosse stato bisogno, il versante politico-istituzionale dei suoi interessi di ricerca: Roberto Ruffilli. Tra il 1970 e il 1973 diverse borse ministeriali furono messe a disposizione di giovani laureati e vari ex-studenti di Prodi iniziarono il proprio curriculum universitario. In quest'ultima data si concluse anche la sua prima esperienza di docente all'Ateneo bolognese, che aveva fatto maturare molte aspirazioni alla ricerca. Poco dopo il trasferimento di Paolo Prodi a Trento, dove fondava l'Istituto storico italo-germanico, si tenne un Convegno a Bagni di Lucca cui parteciparono anche Prosperi e Ruffilli e che fu l'occasione per riflettere sui risultati delle ricerche scaturite da quella belle messe di tesi. Ne venne curato anche il catalogo che, pubblicato, costituì l'efficace testimonianza di una attività didattica intensa, rafforzata dalla presenza di studiosi particolarmente competenti nei rispettivi campi come Prosperi e Ruffilli. Fu soltanto poco tempo dopo la partenza di Prodi che potei apprezzare con maggior consapevolezza le qualità del Maestro e fu in occasione del Convegno si studi $L a$ 
Nascita della Toscana, tenutosi nel 1974 per il $4^{\circ}$ centenario della morte di Cosimo I de' Medici. Ascoltai attentamente gli organizzatori, Furio Diaz e Giorgio Spini, e i singoli relatori, e rimasi sorpresa di sentire dibattere temi e problemi che avevano costituito oggetto delle nostre ricerche cinque o sei anni prima.

Gli eventi che da quella data seguirono nell'Ateneo di Bologna fanno parte della vicenda individuale di ciascuno di quegli allievi. Il gruppo degli studenti di Prodi convisse per qualche tempo in un'unica stanza. La conquista di nuovi spazi segnò anche materialmente il formarsi di nuovi e duraturi equilibri.

Il maestro da quel momento divenne un organizzatore di cultura, affiancandosi nelle nuove esperienze scientifiche nazionali ed internazionali interlocutori come Giuliana Nobili e Piero Schiera che diedero vita con lui ad un Istituto e ad una rivista la cui importanza si può misurare dalla novità delle tematiche proposte e dal numero delle pubblicazioni realizzate. La vivacità di quell'Istituto in cui molti dei presenti si sono più volte incontrati non sarebbe stata pensabile senza il lavoro quotidiano e la competenza scientifica di Giuliana e Piero, vivacità di cui pure sono stata per molti anni testimone. Alle iniziative dell'Istituto storico italo germanico si aggiunsero dal 1975 quelle diverse ed altrettanto vivaci del gruppo interdisciplinare Europa delle Corti, di cui Prodi fu Presidente per dieci anni, che meglio esprimeva nella sua molteplicità e varietà gli interessi iniziali di ricerca del professore, possiamo ora dire bolognese, proiettati anche ad esplorare l'impatto del Concilio di Trento sulle arti figurative, la cultura, la scienza. Ma l'esperienza trentina segnò una svolta nei suoi orientamenti culturali, facendo riaffiorare il primitivo e prioritario interesse per la politica, cui corrispondeva anche l'imperativo etico di un impegno civico perseguito costantemente nelle forme alte della partecipazione alla gestione della cosa pubblica. L'attività di studioso gli era tuttavia più congeniale e, diciamo la verità, gli veniva meglio.

Come Paolo Prodi abbia potuto sostenere un così ampio e variegato numero di impegni e iniziative culturali, non rinunciando alla ricerca originale, né trascurando la didattica, si può comprendere soltanto conoscendo il suo modo di leggere e studiare, che corrisponde anche ad uno stile di vita: leggere attentamente e valutare, trattenere il nuovo e l'utile, selezionare rapidamente, dimenticare quanto non è funzionale alla meta da raggiungere. È in virtù di questa capacità selettiva che di tanto in tanto si chiude in una biblioteca e, con la caparbietà che lo distingue, prende in mano il tema a lungo preparato e accarezzato, e lo sviscera in profondità, arretrando sempre più nel tempo e nello spazio. Il professore ha ben mostrato di meritare quella docenza in Storia che fu una delle ultime non cronologicamente specificata. 
